Esta revista forma parte del acervo de la Biblioteca Jurídica Virtual del Instituto de Investigaciones Jurídicas de la UNAM

\title{
EL NUEVO RÉGIMEN JURÍDICO DE LA RELACIÓN LABORAL DE LOS INVESTIGADORES EN ESPAÑA TRAS LA LEY DE LA CIENCIA: ¿UNA SOLUCIÓN A LA FALTA DE PERSONAL CUALIFICADO?*
}

THE NEW LEGAL REGIMEN IN LABOR RELATIONS IN SPANISH RESEARCHER AFTER THE CIENCE LAW: A SOLUTION DUE TO LACK OF QUALIFIED PERSONNEL?

LE NOUVEAU REGIME JURIDIQUE DES RELATIONS DU TRAVAIL DES CHERCHEURS EN ESPAGNE DERRIERE LA LOI DE SCIENCE: EST-CE QUE C'EST UNE VERITABLE SOLUTION AU MANQUE DE PERSONNEL QUALIFIE?

\section{Lara NOGUEIRA FERREIRO**}

\begin{abstract}
RESUMEN: Con el transcurrir de los años el mercado de trabajo ha sufrido grandes transformaciones impulsadas por el desarrollo tecnológico de los últimos tiempos. El mismo deriva, en parte, de la producción científica generada por un grupo específico de trabajadores cualificados, los investigadores, que, sin embargo, en nuestro país no habían visto modernizada su relación laboral de la misma manera. Por ello, la reciente Ley de la Ciencia trata de fomentar la insuficiente cultura investigadora que hasta el momento existe, haciendo más atractiva la salida profesional del titulado universitario hacia la investigación, con el convencimiento de que el auge en este sector es la solución a los problemas que ha originado la crisis económica y va a evitar su repetición en un futuro. Con este fin, la norma instaura tres nuevas figuras contractuales que pretenden mejorar las condiciones de trabajo de este tipo de personal y su consideración por la so-
\end{abstract}

* Recibido el 16 de febrero de 2016 y aceptado para su publicación el 1o. de abril de 2016. Este trabajo se enmarca en el proyecto de investigación "Análisis jurídico del teletrabajo en el derecho español y comparado. El e-work como modelo de flexibilidad, conciliación y productividad" (DER2013-43615-R), del Ministerio de Economía y Competitividad (España), cuya investigadora principal es la profesora Lourdes Mella.

** Profesora contratada FPU del Ministerio de Educación. 
Esta revista forma parte del acervo de la Biblioteca Jurídica Virtual del Instituto de Investigaciones Jurídicas de la UNAM

ciedad. El presente estudio se centra en analizar si estas modalidades del contrato de trabajo ayudan a alcanzar los objetivos fijados por la norma, salvando con ello el inconveniente de la falta de buenos investigadores dedicados al sector de $\mathrm{I}+\mathrm{D}+\mathrm{i}$ causado por unas desfavorables perspectivas de futuro en el mismo.

Palabras clave: Ciencia, investigador, empleo.

ABSTRACT: Over the years, the labour market has undergone a great transformation driven by the technology development of recent times. It comes, for the most part, from the scientific production generated by a specific group of skilled workers, researchers. However, in our country, they don't see upgrade their labour relation in the same way. For this reason, the Science Act (Act No. 14/2011) seeks to promote the insuffficient scientific culture that exists so far, making more atractive the research career, in the certainty that the boom in this sector is the solution to problems which caused the current crisis and it will avoid their recurrence in the future. For this purpose, the Act establishes three new employment contracts, to improve working conditions of researchers. The current study focuses on the appropriateness of these contracts to achieve the goals set by the law, overcoming the drawback of the skills shortage.

Key Words: Science, researcher, employment.

RÉSUMÉ: Au fil des anées le marché du travail a subi une grande transformation entraînée par le développement de la technologie de ces derniers temps. Il vient, pour la plupart, de la production scientifique généré par un groupe spécifique de travailleurs qualifiés, de chercheurs. Cependant, dans notre pays, ils n'ont pas vu améliorer leurs conditions de travail de la même manière. Pour cette raison, la Loi sur la science (Loi $\mathrm{n}^{\circ}$ 14/2011) vise à promouvoir l'insuffisante culture scientifique qui existe en ce moment, en rendant plus attractive la carrière de recherche, avec la certitude que le boom dans ce secteur est la solution aux problèmes qui ont causé la crise actuelle et il permettra d'éviter qu'elles ne se reproduisent à l'avenir. A cet effet, la Loi établit trois nouveaux contrats de travail, qui s'efforcent d'améliorer les conditions de travail des chercheurs. Cette étude se concentre sur l'adéquation de ces contrats pour atteindre les objectifs fixés par la Loi, pour surmonter l'inconvénient de la pénurie de compétences.

Mots-clés: Science, recherche, emploi. 
Esta revista forma parte del acervo de la Biblioteca Jurídica Virtual del Instituto de Investigaciones Jurídicas de la UNAM

SUMARIO: I. Introducción. II. Una visión general de la situación de los investigadores en España antes de la Ley de Ciencia. III. Los nuevos contratos regulados en la Ley de Ciencia. IV. Conclusiones.

\section{INTRODUCCIÓN ${ }^{1}$}

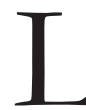

a Ley 14/2011, de 1o. de junio, de la Ciencia, la Tecnología y la Innovación (LCTI), ${ }^{2}$ nace con el claro objetivo, reflejado en su título preliminar, de fomentar la investigación científica y técnica, el desarrollo experimental y la innovación "como elementos sobre los que ha de asentarse el desarrollo económico sostenible y el bienestar social". ${ }^{3}$ Con ella, nuestro país contribuye al impulso de una de las prioridades políticas de la Unión Europea de los últimos años que apunta hacia el aumento del gasto en este sector, cuyo auge promueve la mejora y competitividad de los países miembros. ${ }^{4}$

El preámbulo de la LCTI considera que la generación del conocimiento en todos los ámbitos, su difusión y aplicación para la obtención de un beneficio social o económico son actividades esenciales para el progreso de nuestra sociedad. De este modo, la inversión en investigación es una de las claves para que el país se recupere de la crisis en la que está inmerso. Cabe afirmar que los puestos de trabajo se generan por la creación de empresas, y éstas nacen motivadas por la exigencia de dar respuesta a las necesidades de potenciales clientes ofreciéndoles un objeto o prestación diferenciado, que sólo puede ser

1 Abreviaturas: LCTI (Ley 14/2011, de 1 de junio, de la Ciencia, la Tecnología y la Innovación), I+D+i (investigación, desarrollo e innovación), FPU (ayudas a la Formación de Profesorado Universitario), IF (investigador en formación), IFI (investigador en fase inicial), FJI (Fundación Jóvenes Investigadores-Precarios), IE (investigador experimentado), EBI (Estatuto del Becario de Investigación), EPIF (Estatuto del Personal Investigador en Formación), BOE (Boletin Oficial del Estado), RD (real decreto), EBI (Real Decreto 1326/2003, de 24 octubre, por el que se establece el Estatuto del Becario de Investigación), EPIF (Real Decreto 63/2006, de 27 enero, por el que se promulgaba el Estatuto del Personal Investigador en Formación), STS (sentencia de la Sala de lo Social del Tribunal Supremo), SECTI (Sistema Español de Ciencia, Tecnología e Innovación), ET (Real Decreto Legislativo 1/1995, de 24 de marzo, por el que se aprueba el texto refundido de la Ley del Estatuto de los Trabajadores), SMI (salario mínimo interprofesional), LOU (Ley Orgánica 6/2001, de 21 de diciembre, de Universidades).

2 Publicada en el Boletín Oficial del Estado (BOE), núm. 131, del 2 de junio de 2011.

3 Artículo 1o. de la LCTI.

4 Este objetivo se ha plasmado en la estrategia europea 2020, cuyo texto completo se encuentra disponible en: bttp:/ / ec.europa.eu/ europe2020/index_es.htm. 
Esta revista forma parte del acervo de la Biblioteca Jurídica Virtual del Instituto de Investigaciones Jurídicas de la UNAM

creado gracias a un trabajo de investigación. Así, el dedicar nuestros recursos en $\mathrm{I}+\mathrm{D}+\mathrm{i}$ es la vía adecuada para que se pueda competir con países emergentes, o los ya consolidados, mediante productos, procesos o servicios que sólo nosotros podamos brindar.

De esta forma, el estímulo de la actividad investigadora es posible dirigirlo hacia dos puntos: el primero, los organismos que desarrollan una actividad innovadora y, el segundo, los medios humanos que permiten llevar a cabo esta clase de tareas. En ambos aspectos incide una de las principales novedades de la citada norma, la regulación de tres modelos contractuales creados para: 1) promover la inversión en el sector dotando de reconocimiento y prestigio a las actividades investigadoras; 2) determinar el régimen jurídico del personal investigador facilitando el proceso de reclutamiento y aumentando el interés por las contrataciones laborales en este ámbito, y 3) hacer más atractiva la carrera profesional cumpliendo con las necesidades de un personal cualificado, procurando captar a científicos de excelencia que puedan situar a España como país de referencia.

Por lo tanto, el presente estudio se centra en analizar si estas nuevas modalidades del contrato de trabajo son suficientes para alcanzar las citadas pretensiones o si, por el contrario, existen aspectos en la nueva regulación jurídica que no han mejorado lo suficiente para que la salida laboral hacia la investigación se considere preferente frente a cualquier otra. La metodología utilizada comienza por dar una ojeada básica a la situación anterior a la LCTI para, posteriormente, observar las nuevas condiciones laborales fijadas por la Ley para los investigadores en cada uno de los estadios de su carrera profesional.

\section{UNA VISIÓN GENERAL DE LA SITUACIÓN DE LOS INVESTIGADORES EN ESPAÑA ANTES DE LA LEY DE CIENCIA}

\section{En la etapa predoctoral}

El tratamiento jurídico de los investigadores en formación (IF) o en fase inicial (IFI) ${ }^{5}$ ha sido tema de debate en nuestro país desde su aparición, puesto

5 La Fundación Jóvenes Investigadores-Precarios (FJI) emplea de forma habitual este último término extraído del programa de becas de investigación Marie Slodowska-Curie, concretamente de su apartado Personas. El mismo flexibiliza la figura del investigador en formación, 
Esta revista forma parte del acervo de la Biblioteca Jurídica Virtual del Instituto de Investigaciones Jurídicas de la UNAM

que, hasta hace bien poco, éstos habían estado desprovistos de regulación jurídica, siendo potestad de las universidades u otros organismos de investigación en los que se integraban, la fijación de sus condiciones de acceso y prestación de servicios. ${ }^{6}$ Habitualmente, el vínculo se concebía bajo la apariencia de una beca, negando la existencia de relación laboral por parte de las instituciones otorgantes. Esta etapa se caracterizaba por un alto grado de precariedad, ${ }^{7}$ ya que, entre otras carencias, a sus integrantes se les apartaba de la mínima protección social.

Con los años, en atención a las exigencias del grupo, se emprendió un proceso de dignificación y laboralización del que informan dos normas: 1) el Real Decreto 1326/2003, de 24 octubre, por el que se establecía el Estatuto del

puesto que no sólo atiende a la obtención o no del grado de doctor, sino que la principal diferencia se basa en los años de experiencia profesional como investigador. De este modo, aquel que haya investigado durante menos de cuatro años (o cinco, en ocasiones) se puede denominar investigador en fase inicial y, por el contrario, tras la superación de este límite, la expresión utilizada es la de investigador experimentado (IE). Para el presente estudio se opta por utilizar el término investigador en formación, puesto que ése es el utilizado por la normativa contemplada, a pesar de que para ciertas afirmaciones, quepa indicar lo innecesario del título.

6 Moreno Gené, J., "El contrato predoctoral: luces y sombras en la contratación del personal investigador en formación tras la nueva Ley de la Ciencia", Impacto de la nueva legislación en la educación superior y la investigación, Sevilla, Secretariado de Publicaciones de la Universidad de Sevilla, 2012, p. 246; encomia la figura alegando que realiza "gran parte de la producción científica de este país".

7 Así la califican, entre otros, Serrano Argüello, N., "Modalidades específicas de contratación laboral del personal investigador de la precarización al desiderátum de su estabilidad en el empleo y consolidación profesional", Revista General de Derecho del Trabajo y de la Seguridad Social, núm. 36, 2014, p. 131; Cristobal Roncero, R., "El personal investigador en formación: un contrato común con peculiaridades", Revista del Ministerio de Trabajo e Inmigración, núm. 83, 2009, p. 351; Moreno Gené, J., "El personal investigador en formación ¿becarios o trabajadores?”, Temas Laborales: Revista Andaluza de Trabajo y Bienestar Social, núm. 78, 2005, p. 133; id., "La contratación laboral de los investigadores en la Ley de la Ciencia, la Tecnología y la Innovación”, Estudios financieros. Revista de Trabajo y Seguridad Social. Comentarios, Casos Prácticos: Recursos Humanos, núm. 340, 2011, p. 84; Angel Guardia, M., "Cambios en la gestión de la investigación”, Impacto de la nueva legislación en la educación superior y la investigación, Sevilla, Secretariado de Publicaciones de la Universidad de Sevilla, 2012, p. 55.

Además, la FJI incluye en su nombre completo la calificación de "precarios", siendo uno de sus cometidos trabajar por la eliminación de la precariedad laboral en la que están inmersos muchos investigadores, tal y como afirman en su Informe sobre la carrera investigadora (ICI) de

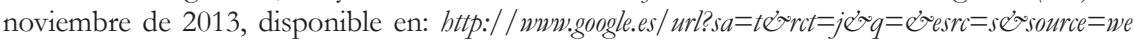
$b \& c d=1 \& v e d=0 C C E Q F j A A \& u r l=b t t p \% 3 A \% 2 F \% 2 F$ precarios.org $\% 2 F I n f o r m e \% 2 B$ Carrera $\% 2 B I$ nvestigadora\%2B2013\&ei=b46BVfPXLcGsygOvYGwDA\&usg= AFQjCNGUMUyKWLkMcj88I Fa_Unyk_4HZ7A\&bum=bv.96041959,d.ZGU. 
Esta revista forma parte del acervo de la Biblioteca Jurídica Virtual del Instituto de Investigaciones Jurídicas de la UNAM

Becario de Investigación (EBI), que afirmaba la naturaleza jurídica administrativa para el periodo formativo posterior a la consecución de la suficiencia investigadora de aquellos beneficiarios de una beca, concedida en virtud de programas de ayudas inscritos en el registro de becas de investigación, y 2) el Real Decreto 63/2006 de 27 enero, por el que se promulgaba el Estatuto del Personal Investigador en Formación (EPIF), que optaba por un modelo mixto de beca, durante los dos primeros años de la carrera, y de contrato en prácticas, los dos siguientes, para los beneficiarios de programas de ayuda dirigidos al desarrollo de actividades de formación y especialización científica y técnica a través, como mínimo, de los correspondientes estudios oficiales de doctorado. Durante el tiempo de beca, ambas normativas contemplaban su asimilación a los trabajadores por cuenta ajena, quedando así protegidos en la mayoría de contingencias posibles, ${ }^{8}$ y mejorando la situación de la que disfrutaban hasta entonces. ${ }^{9}$

8 Se trata, en todo caso, de una inclusión parcial. Ciertos beneficios como puede ser la prestación por desempleo, no van a ser nunca de aplicación a los becarios de investigación, siendo necesaria la firma de un contrato de trabajo para que se entiendan protegidos por esta contingencia. Al respecto, véanse Aguilera Izquierdo, R., "El Estatuto del Becario de Investigación”, Revista Española de Derecho del Trabajo, núm. 121, 2004, pp. 27-43; García Ninet, J. I., "Sobre el presunto Estatuto del Becario de Investigación", Tribuna Social, núm. 155, 2003, pp. 5-10; García Valverde, M., "Naturaleza de la relación que une al «supuesto becario» con la universidad”, Aranzadi Social, núm. 12, 2005, pp. 2468-2474; Hierro Hierro, F. J., “Acerca de la protección social dispensada al becario de investigación por el RD 1326/2003, de 24 de octubre", Anuario de la Facultad de Derecho (Universidad de Extremadura), núm. 22, 2004, pp. 293305; Luján Alcaraz, J., "A propósito del Estatuto del Becario de Investigación”, Aranzadi Social, núm. 1, 2004, pp. 23-34. No obstante, el Real Decreto 1493/2011, de 24 octubre, por el que se regulan los términos y las condiciones de inclusión en el Régimen General de la Seguridad Social de las personas que participen en programas de formación, en desarrollo de lo previsto en la disposición adicional tercera de la Ley 27/2011, de 1 de agosto, sobre actualización, adecuación y modernización del Sistema de la Seguridad Social y el Real Decreto-Ley 5/2013, de 15 marzo, de medidas para favorecer la continuidad de la vida laboral de los trabajadores de mayor edad y promover el envejecimiento activo, han previsto la posibilidad de que aquellos que hayan participado en programas de formación, sin haber recibido ninguna cobertura en materia de seguridad social, puedan recuperar, en parte, los periodos de cotización sin aportación. Sobre ello profundizan Carrizosa Prieto, E., "La protección social del personal docente e investigador en formación: el convenio especial de la seguridad social", Aranz̧adi Social, vol. 5, núm. 5, 2012, pp. 161-180; Moreno Gené, J., "La cotización de los antiguos becarios tras el Real Decreto-Ley 5/2013”, XXXII Jornadas Universitarias Andaluzas de Derecho del Trabajo y Relaciones Laborales: Retos del Derecho del Trabajo frente al desempleo juvenil, Granada, 21 y 22 de noviembre de 2013.

9 Es muy crítico Duque González, M., Becas y becarios: camino de su regulación laboral, Valladolid, Lex Nova-Thomsons Reuters, 2012, p. 146, con la justificación del periodo de beca en estas 
Esta revista forma parte del acervo de la Biblioteca Jurídica Virtual del Instituto de Investigaciones Jurídicas de la UNAM

La sustitución progresiva de becas por contratos laborales se sustentaba en la noción de que primar la finalidad formativa para justificar un periodo de beca en estos casos no era correcto, ${ }^{10}$ como se refleja en la Carta Europea del Investigador y el Código de conducta para la contratación de investigadores del 11 de marzo de 2005, ${ }^{11}$ cuyas indicaciones aconsejaban su contratación desde la aceptación en el programa de doctorado, punto de inicio de la carrera investigadora.

De hecho, la realidad de los investigadores en formación no ha cambiado sustancialmente conforme ha ido avanzando su regulación, por lo menos en lo que se refiere a su actividad diaria. Así, cabe recordar que se considera IF al que comienza su carrera investigadora mediante su admisión a un programa de doctorado y, cuyo fin, se alcanza por la presentación de un trabajo original de investigación, que lo hace merecedor del título de doctor y lo capacita "para el trabajo autónomo en el ámbito de la I+D+i". ${ }^{22}$ Ante esto,

situaciones, pues considera que se "está echando por tierra todo el sistema educativo español" al permitir dar "validez nula a un título académico de la envergadura de una licenciatura" para justificar la adscripción a una beca asegurando "que la persona que lo ostenta carece de formación suficiente para desempeñar un puesto de trabajo".

10 A la hora de calificar la relación que mantiene un IF con su entidad de adscripción (aquella en la que desempeña su labor) la jurisprudencia se aferra a la teoría del interés predominante. La misma consiste en determinar qué prevalece como utilidad de la beca, si la formación que recibe el investigador o la actividad que este presta a favor de la entidad. Así, ciertos aspectos como el beneficio, fruto de su trabajo, se acumule al patrimonio del otorgante de la beca o, que otro trabajador hubiera tenido que realizar las tareas de las que se encarga el becario para el buen funcionamiento de la organización, indicarían que predomina la utilidad de la institución y, por tanto, la relación habría de ser calificada como laboral. Entre otras, así lo entiende la Sentencia de la Sala de lo Social del Tribunal Supremo (STS) del 29 de marzo de 2007 (RJ 2007\3191). En este aspecto profundizan, Duque González, M., Becas y becarios..., cit., pp. 58-62; Fernández Díaz, P., Los contratos de trabajo formativos en prácticas, Valencia, Tirant lo Blanch, 2014, pp. 89-100; Morón Prieto, R., "Movilidad normativa, crisis económica y crisis de la autonomía en la gestión del personal docente e investigador universitario", Conclusiones XII curso de régimen jurídico de universidades, Navarra, Aranzadi-Thomson Reuters, 2014, p. 91.

11 2005/251/CE.

12 Artículo 13 del RD 99/2011, del 28 de enero, por el que se regulan las enseñanzas oficiales de doctorado. La dedicación a actividades de investigación no se prohíbe por no obtener el doctorado, al igual que tampoco se limita la dirección de una empresa sino se ha cursado el grado en administración y dirección de empresas, y al contrario de ciertos ámbitos profesionales, como el ejercicio de la abogacía, que sí se veta a los graduados en derecho. No obstante, en las universidades las instituciones que más investigadores acogen, la titulación sí es preceptiva para la suscripción del los contratos de ayudante doctor y contratado doctor y, además, para la asunción de ciertas responsabilidades, como la docencia de asignaturas completas o la dirección de tesis. Pese a esto, para aquellos que ejercen actividades de investigación al margen de 
Esta revista forma parte del acervo de la Biblioteca Jurídica Virtual del Instituto de Investigaciones Jurídicas de la UNAM

cabe tener en cuenta que la elaboración de una tesis no es un trabajo menor sino que se trata, desde su comienzo, de una profunda investigación del tema elegido. Probablemente, para algunos de los que emprenden este camino, se trata de su primer contacto con el sector de la investigación, ${ }^{13}$ pero a pesar de ello, su periodo de formación no debiera exceder de unos pocos meses, toda vez que el doctorando ya posee una titulación universitaria y conocimientos mínimos en el área de estudio. ${ }^{14}$ De lo contrario, si se entendiera que la mayor parte del tiempo — durante los cuatro años habitualmente utilizados para el desarrollo de la tesis_ - el investigador se está únicamente formando, la culminación de ésta no sería posible, puesto que la necesidad de tan dilatada formación indicaría una gran falta de preparación que le imposibilitaría llevar a cabo la investigación, objeto del programa de doctorado. Además, el cambio de titulaciones al que se somete nuestro sistema educativo, concretamente la implantación del plan Bolonia y la instauración de los grados universitarios, implica ya una primera toma de contacto con el campo de la investigación, puesto que para obtener el título de graduado y de máster, es necesario presentar un trabajo final de grado de inexcusable carácter innovador, lo que reduce al mínimo las necesidades formativas del sujeto que ya ha obtenido estas credenciales.

De esta manera, mantener la opción por la relación de beca se ha de considerar difícilmente sustentable, ya que, aunque la finalidad principal del periodo de doctorado es conseguir la titulación de doctor, la actividad por la que éste se consigue no difiere de la que posteriormente ha de desempeñar si continúa con su carrera investigadora. La doctrina avala también este punto de vista, al considerar que la actividad investigadora que realiza este personal "tiene desde su inicio la suficiente entidad para ser considerada como una actividad productiva más allá de la formación que se recibe". ${ }^{15}$

un programa de doctorado, el artículo 20.5 y la disposición adicional vigesimoctava de la LCTI también obliga a su contratación, siempre que la entidad empleadora cumpla ciertos requisitos.

13 En una gran parte de las titulaciones universitarias de diplomatura o licenciatura (ahora a extinguir) no se exigía la elaboración de ningún tipo de trabajo de investigación o innovador para la consecución del grado que se había cursado. Sin embargo, no era poco habitual que los docentes utilizaran esta herramienta como parte de su sistema evaluativo.

14 El citado RD 99/2011, en su artículo 6o. establece las diferentes titulaciones que dan acceso a un programa oficial de doctorado.

15 Así lo considera en sus obras Moreno Gené, J., "El contrato predoctoral: luces...", op. cit., p. 245; "La contratación laboral de los investigadores...", op. cit., p. 85; también defienden 
Esta revista forma parte del acervo de la Biblioteca Jurídica Virtual del Instituto de Investigaciones Jurídicas de la UNAM

\section{En la etapa postdoctoral}

Por su parte, una vez obtenido el grado de doctor y, por tanto, superada la fase inicial en el ámbito científico, las perspectivas laborales no tornaban más halagüeñas. De hecho, la cobertura legislativa para este periodo de la carrera profesional ha ido transitando en paralelo al detallado para los IF.

Así, en un primer momento la nota característica era la ausencia de cualquier tipo de regulación que aclarara las condiciones laborales de los doctores que continuaban prestando servicios de $\mathrm{I}+\mathrm{D}+\mathrm{i}$. Por ello, lo más habitual era el recurso a becas de investigación postdoctorales cuya finalidad consistía en el perfeccionamiento y ampliación de los conocimientos adquiridos en la fase predoctoral. Habitualmente, este tipo de ayudas se planteaban para dar la posibilidad al beneficiario de seguir desempeñando su labor en el extranjero, como complemento a los estudios que acababa de superar. No obstante, también han existido convocatorias cuyo disfrute se limitaba a instituciones nacionales o, simplemente, a alguna distinta de la que hubiera obtenido el título de doctor.

La falta de una normativa general hacía necesario - al igual que para los predoctorales - que fueran los textos que invocaban las ayudas los que completaran el vacío legal. Aún así, lo habitual era que estos fijaran de un lado, el carácter no laboral de la relación y de otro, los requisitos de acceso de los beneficiarios y de sus centros de adscripción, delegando en estos últimos la carga de establecer derechos y deberes propios del investigador. Todo ello generaba un gran problema de desigualdad, puesto que dos doctores podían estar ejerciendo el mismo puesto con condiciones totalmente diferentes (salario, jornada, vacaciones, etcétera) tan sólo por pertenecer a dos entidades distintas.

Las características de estas ayudas no diferían demasiado de las que se ocupaban del periodo predoctoral, salvo su duración inferior y la necesidad de que el solicitante hubiera conseguido el título de doctor recientemente. Con esto último se justificaba la supuesta finalidad formativa de la prestación de servicios, para seguir empleando la figura del becario en base a su escasa experiencia y, por tanto, a la necesidad de seguir formándose. Sin embargo, si ya era difícil sostener esta idea para aquellos que cursaban estudios de tercer ciclo, mayor

la relación laboral, Cristóbal Roncero, R., op. cit., pp. 331 y 332; Fernández Díaz, P., op. cit., p. 40, que se muestra partidaria a centrar los esfuerzos de la administración en aquellas alternativas a las becas. 
era la dificultad para este grupo, por lo que los argumentos utilizados a favor de esta opción podían resultar un tanto artificiosos e interesados. Así, se conseguía eternizar esta figura, que permitía seguir explotando las cualidades del investigador — ahora titulado — sin sobrecoste alguno para las instituciones que lo acogían. Lógicamente, todo ello redundaba en perjuicio del beneficiario que se perpetuaba en una situación de desprotección social, siendo en ocasiones incluso más penosa que la del doctorando, pues ni siquiera el régimen especial de estudiantes le podría ser aplicado. ${ }^{16}$

Más adelante, tras la promulgación del EBI, se adoptaba una solución parcial al problema, considerando trabajadores por cuenta ajena a los beneficiarios de una ayuda postdoctoral a efectos de seguridad social. Así, éstos quedaban protegidos ante cualquier contingencia, salvo el desempleo; no obstante, la necesidad de que los beneficiarios lo fueran respecto a una beca registrada en el registro de becas de investigación, dejaba al margen de la protección social a una buena parte de doctores. Afortunadamente, en los años siguientes se aprobó el EPIF y, aunque por su nombre parecía dejar al margen la figura de los doctores, procedía a establecer una mejora categórica para el grupo, la obligación de su contratación laboral por parte de las entidades de adscripción. ${ }^{17}$ Por lo tanto, aquí culminaba el proceso de laboralización de los beneficiarios de ayudas a la investigación con el título de doctor.

\section{Tras una dilatada experiencia}

El tercer estadio de la carrera investigadora del que se va a ocupar la LCTI es aquella etapa en la que el bagaje profesional del científico lo sitúa como un referente en su ámbito. Se trata de una figura de la que la normativa no se ha ocupado demasiado, quizás, porque se considera marginal en relación a otras modalidades contractuales que se utilizan en la investigación. Asimismo, existe también la percepción de que este colectivo no necesita de tanta pro-

16 Moreno Gené, J., "La laboralización de los investigadores postdoctorales", CEF. Trabajo y Seguridad Social. Comentarios y casos prácticos, núm. 304, 2008, p. 50.

17 Disposición adicional sexta del EPIF. Para su contratación se podían emplear los distintos modelos contractuales del Estatuto de los Trabajadores y, si el centro de adscripción era un organismo público de investigación, también era posible la utilización de los contratos establecidos por la derogada Ley 13/1986, de 14 de abril, de Fomento y Coordinación General de la Investigación Científica y Técnica. 
Esta revista forma parte del acervo de la Biblioteca Jurídica Virtual del Instituto de Investigaciones Jurídicas de la UNAM

tección como la de aquellos que se inician en la investigación, toda vez que, gracias a la experiencia que ostentan, se presume que no presentan problemas de inserción laboral.

Por lo que no existen antecedentes normativos respecto de la relación (laboral o no) entre un investigador experimentado y el centro de adscripción, salvo el modelo de contrato aplicable a las universidades, actualmente denominado de profesor visitante, ${ }^{18}$ que también se dirige a la contratación de expertos en un determinado ámbito. La característica que se puede extraer de esta figura es la flexibilidad con la que cuenta, puesto que sus suscriptores pueden acordar los aspectos que consideren sin prácticamente ninguna limitación.

\section{LOS NUEVOS CONTRATOS REGULADOS EN LA LEY DE CIENCIA}

\section{El contrato predoctoral}

El contrato predoctoral ha sido ideado específicamente para el investigador en etapa formativa, en atención a sus especiales características. Por lo tanto, culmina el proceso de laboralización, pero no así el de dignificación de este grupo de trabajadores, puesto que, como veremos a lo largo del estudio, las nuevas condiciones profesionales de éstos no son siempre las más deseables.

Los sujetos que pueden suscribir este tipo de contrato son de la parte del trabajador, aquellos que, en primer lugar, ostenten el título de licenciado, ingeniero, arquitecto, graduado universitario o máster universitario y, en segundo lugar, hayan sido admitidos en un programa de doctorado. Su ocupación atañe la realización de tareas de investigación en el ámbito de un proyecto específico y novedoso, finalidad del contrato predoctoral. ${ }^{19}$ Por lo tanto, y aunque la norma no lo especifique, este proyecto al que se ha de dedicar el contratado es, lógicamente, la tesis doctoral, la cual una vez elaborada, defendida y aprobada permite la obtención del título de doctor. Por otro lado, la

18 La Ley 14/1970, de 4 de agosto, General de Educación y Financiamiento de la Reforma Educativa, en su artículo 120 permitía a las universidades contratar, por tiempo limitado, a profesores españoles o extranjeros en consideración a su prestigio y méritos reconocidos para atender a campos de especialización restringida. Asimismo, en el mismo artículo se establecía la posibilidad de contratar a aquellos de excepcional prestigio de manera indefinida, si sus servicios se consideraban necesarios.

19 Artículo 21, inciso a, de la LCTI. 
Esta revista forma parte del acervo de la Biblioteca Jurídica Virtual del Instituto de Investigaciones Jurídicas de la UNAM

parte contratante se limita a los organismos públicos de investigación de la administración general del Estado, organismos de investigación de otras administraciones públicas y a universidades públicas cuando sean perceptoras de fondos cuyo destino incluya la contratación de personal investigador, o, para el desarrollo de sus programas propios de I + D + i. ${ }^{20}$ No obstante, esta breve enumeración de entidades empleadoras legitimadas para la firma del contrato predoctoral se amplía considerablemente mediante la disposición adicional primera de la Ley, incluyendo a las universidades privadas y de la Iglesia católica ${ }^{21}$ perceptoras de fondos cuyo destino incluya la contratación de personal investigador; los consorcios públicos y fundaciones del sector público participadas por el Estado, cuyo fin u objeto social comprenda la ejecución directa de actividades de investigación científica y técnica o de prestación de servicios tecnológicos o, aquellas otras de carácter complementario necesarias para el adecuado progreso científico y tecnológico de la sociedad. Estas actividades deben formar parte de los programas de desarrollo del Plan Estatal de Investigación Científica y Técnica o del Plan Estatal de Innovación, y otros organismos de investigación de la administración general del Estado diferentes de los organismos públicos de investigación cuando realicen actividad investigadora y sean beneficiarios de ayudas o subvenciones públicas que incluyan en su objeto la contratación de personal investigador. Además, los centros pertenecientes al sistema nacional de salud, y los vinculados o concertados con él, que desempeñen cierta actividad investigadora, pueden contratar de este modo a su personal, considerándose por ello personal investigador. ${ }^{22}$

Como se puede observar, los entes públicos ocupan una posición transcendental, puesto que a ellos, como empleadores, está dirigida principalmente esta modalidad contractual, y porque además, suelen ser los órganos competentes para otorgar los fondos o ayudas que legitiman el uso del contrato predoctoral a otra clase de entidades. Ciertamente, en las universidades públicas y otros organismos públicos de investigación es donde se desarrollan

20 Artículo 20.2 de la LCTI.

21 La diferenciación específica entre la universidad privada y de la Iglesia católica no es necesaria, toda vez que la Ley Orgánica 6/2001, de 21 diciembre de Universidades (LOU) somete la segunda al régimen propio de la primera. Véase Martínez López-Muñiz, J. L., "Las universidades privadas en la Ley de la Ciencia”, Ciencia, tecnología e innovación. Nuevo régimen jurídico, Granada, Comares, 2013, p. 161.

22 Disposición adicional 4a. de la LCTI. 
Esta revista forma parte del acervo de la Biblioteca Jurídica Virtual del Instituto de Investigaciones Jurídicas de la UNAM

las tesis doctorales y, por lo tanto, donde se concentra el núcleo principal de las actividades investigadoras desarrolladas en el país. ${ }^{23} \mathrm{~A}$ pesar de ello, resulta criticable apartar a otras entidades con ánimo de lucro o a las empresas privadas del uso de este tipo contractual. Estos organismos también realizan una importante tarea investigadora y son, en múltiples ocasiones, protagonistas de descubrimientos y adelantos tecnológicos que pueden situar a España en un puesto aventajado respecto de su producción innovadora. De hecho, el preámbulo de la LCTI promueve que la innovación se incorpore definitivamente como una actividad sistemática de toda empresa, sin importar su sector, y requiere la colaboración de ellas, incluso mediante aportaciones económicas. Concretamente, la norma destaca "el protagonismo de las empresas en el ámbito del desarrollo tecnológico y la innovación” por ser éstas fundamentales "para transformar la actividad de investigación científica y técnica en mejoras de la productividad española y de la calidad de vida de los ciudadanos". Como se ha expuesto, los recursos empresariales empleados en investigación son superiores a los de los entes públicos, por ello, el fomento de estas actividades privadas es esencial. Con base en esto, el permitir el uso del contrato predoctoral en la empresa es un modo de aumentar su potencial innovador, facilitando también que estas puedan ser beneficiarias de las ayudas que subvencionan este tipo de contratos y, además, facilita la incorporación del IF al mercado de trabajo.

Por otro lado, la necesidad de que bajo esta modalidad el contratado sea admitido en un programa de doctorado no debe ser una limitación para permitir su uso en el sector privado. Esto permite la cooperación entre los diferentes agentes que conforman el Sistema Español de Ciencia, Tecnología e Innovación (SECTI) si se consintiera la realización de actividades investigadoras en el ente privado conjuntamente con un programa de doctorado a cursar, por ejemplo, en una universidad pública, ${ }^{24}$ de igual manera que en titulaciones inferiores se facilita la realización de prácticas externas al organismo que imparte docencia. Además, nada impide establecer un contrato predoctoral entre la empresa privada e investigador en formación coetáneo a la admisión

23 Moreno Gené, J., "El contrato predoctoral: una modalidad específica de contrato formativo para los investigadores", Aranz̧adi Social: Revista Doctrinal, vol. 5, núm. 3, 2012, p. 11 (versión web Aranzadi).

24 Entiéndase también en las menciones posteriores, referido a cualquier organismo habilitado para impartir un programa de doctorado. 
Esta revista forma parte del acervo de la Biblioteca Jurídica Virtual del Instituto de Investigaciones Jurídicas de la UNAM

del mismo en un programa de doctorado de cualquier Universidad. Todo ello, sin perjuicio de que se apliquen en estas entidades las medidas de control necesarias que garanticen la correcta gestión de este tipo de contratos y, más intensamente, de las ayudas de las que puedan ser beneficiarios. ${ }^{25}$

De la misma manera, este contrato ha de ser celebrado por escrito ${ }^{26}$ y a jornada completa, entre el personal investigador predoctoral en formación, en su condición de trabajador, y la universidad pública u organismo de investigación titular de la unidad investigadora, en su condición de empleador. Contrato que se debe acompañar del escrito de admisión al programa de doctorado expedido por la unidad responsable de dicho programa, o por la escuela de doctorado o posgrado, en su caso. Su duración no puede ser inferior a un año ni exceder de cuatro y, en el caso de haber sido contratado por menos de cuatro años, se pueden concertar prórrogas de un mínimo de doce meses. La actividad del IF se ha de evaluar anualmente por la comisión académica del programa de doctorado o de la escuela de doctorado, siendo causa de rescisión del contrato una evaluación negativa. En ningún caso, el trabajador puede estar contratado mediante esta modalidad durante más de cuatro años, en la misma o distinta entidad, salvo que éste sea discapacitado y las características de la actividad investigadora y el grado de sus limitaciones en la actividad hagan necesario su postergación hasta los seis años, incluyendo las prórrogas. ${ }^{27} \mathrm{~A}$ este respecto, cabe destacar que se percibe una exagerada temporalidad del contrato predoctoral. Por supuesto, la finalidad del

25 Asimismo, esta relación simultánea entre trabajador, empresa privada y administración pública se permite y favorece en la norma respecto de la movilidad de los investigadores pertenecientes a los organismos públicos. Véase artículo 17 de la LCTI.

26 De nuevo se trata de una previsión innecesaria. El artículo 8.2 del ET, de aplicación supletoria en estos casos, ya establece la forma escrita para todos los contratos temporales superiores a cuatro semanas, pues de lo contrario se entiende celebrado de forma indefinida a tiempo completo.

27 Artículo 21, letras b) y c). A propósito de las prórrogas del contrato predoctoral, Moreno Gené, J., "El contrato predoctoral: luces...”, op. cit., p. 16, considera aplicable la prórroga automática fijada por el artículo 49.1, inciso c) del ET, cuando no existe denuncia por ninguna de las partes y el trabajador sigue prestando servicios. Sin embargo, la aplicación supletoria implica que la norma principal no regule el aspecto en cuestión y, en este caso, el procedimiento para prorrogar el contrato sí está establecido. De esta manera, en caso de no existir denuncia pero tampoco informe favorable, se incumple uno de los requisitos que permiten prorrogar la relación laboral y, en mi opinión, la misma se ha de dar por finalizada. En el mismo sentido, Serrano Argüello, N., "Los contratos laborales del personal investigador", Ciencia, tecnología e innovación. Nuevo régimen jurídico, Granada, Comares, 2013, p. 96. 
Esta revista forma parte del acervo de la Biblioteca Jurídica Virtual del Instituto de Investigaciones Jurídicas de la UNAM

mismo implica necesariamente que este modelo contractual sea temporal, ya que no puede prolongarse más allá del tiempo necesario para la elaboración de la tesis.

No obstante, la necesidad de un informe favorable anual para la subsistencia de la relación laboral puede parecer excesiva. En algún tipo de actividades investigadoras el avance que se percibe año tras año es escaso, y tener que probarlo anualmente puede ser una tarea ardua que derive en cierta inseguridad para el trabajador e, incluso, para el empleador. De esta manera, quizás sea suficiente la emisión de un informe cada dos años, como se realizaba hasta el momento, para justificar las ayudas a la formación de investigadores.

Por su parte, en caso de incapacidad temporal, riesgo durante el embarazo, maternidad, adopción o acogimiento, riesgo durante la lactancia y paternidad se suspende el cómputo de la duración del contrato. Asimismo, su finalización puede darse: por expiración del año de duración en caso de informe desfavorable de la comisión académica del programa de doctorado o de la escuela de doctorado, por haber obtenido el título de doctor ${ }^{28}$ o por haberse cumplido la duración máxima de cuatro o seis años, independientemente de que se haya concluido o no el doctorado.

La retribución de estos trabajadores no puede ser inferior al 56\% del salario fijado para las categorías equivalentes en los convenios colectivos de su ámbito de aplicación durante los dos primeros años, al 60\% durante el tercer año, y al 75\% durante el cuarto año. Tampoco puede ser nunca inferior al salario mínimo interprofesional (SMI) que se establezca anualmente. ${ }^{29} \mathrm{El}$ primer problema que se nos plantea es establecer cuál es la categoría equivalente al investigador predoctoral en formación. Cabe pensar que, a estos efectos, se ha de tener en cuenta el salario de un investigador doctor, puesto que a esa

28 En contra, Moreno Gené, J., "La contratación laboral de los investigadores en la Ley de la Ciencia, la Tecnología y la Innovación: ¿el final del estado de excepción laboral en la ciencia?”, Revista de Trabajo y Seguridad Social: Comentarios, casos prácticos, núm. 340, 2011, p. 96, que determina que "al estar configurado como un contrato a término", la obtención del doctorado "no debe suponer automáticamente la extinción del contrato predoctoral", terminando éste cuando "finalice la duración prevista en el contrato inicial o en sus prórrogas". A pesar de esto, las ayudas que fomentan este modelo contractual establecen la finalización del mismo tan pronto como se obtenga el título de doctor.

29 Artículo 21, inciso d, de la LCTI. Tampoco era necesaria esta afirmación, ya que un contrato a tiempo completo nunca podrá ser retribuido por debajo del SMI al amparo del artículo 27 del ET. 
categoría se dirige la carrera del predoctoral y por cuya situación (no poseer el título de doctor) ya se realiza un descuento en estas retribuciones, sin necesidad de acudir a un salario menor. En segundo lugar, la cantidad retributiva no parece suficiente para fomentar la captación de talento, aunque quizá sí lo sea en un primer momento para animar a los empleadores a utilizarlo debido a su menor coste salarial, y así fomentar inicialmente la actividad investigadora. A pesar de ello, si la parte contratante no es capaz de atraer y retener con ese salario al investigador excelente, difícilmente puede despuntar en el terreno investigado, $\mathrm{y}$ al final, el gasto que ocasiona el traspaso de un trabajador a otro de manera continua es mayor. Cabe considerar que este contrato es el peor remunerado de todos los existentes, al menos en sus dos primeros años, es decir, cualquier otro modelo contractual genera más beneficios al trabajador que el aquí estudiado.

Con todo, el efecto más favorable de la contratación laboral del investigador predoctoral en formación es su inclusión en el Régimen General de la Seguridad Social como trabajador por cuenta ajena. Desde la firma del contrato, este colectivo goza de una protección social total, incluida la contingencia de desempleo, por la cual se cotizaba tan sólo los dos años de vigencia del contrato en prácticas. Ahora bien, la eficacia de este cambio depende de la base de cotización aplicada al empleado en función del salario que finalmente le sea asignado. De otro lado, las entidades que deciden utilizar este tipo de contratación se benefician de una reducción del 30\% de la cuota empresarial a la seguridad social por contingencias comunes en la cotización relativa a este colectivo, ${ }^{30}$ lo que funciona como incentivo para aquellas entidades que suscriban contratos de este tipo, además de paliar el incremento de coste del trabajador, por el cambio de beca (cuando la cantidad a cotizar era mínima) a contrato.

Por último, cabe destacar que el desarrollo del régimen del investigador en formación debe realizarlo el gobierno mediante un nuevo Estatuto del personal investigador en formación, en un plazo de dos años desde la entrada en vigor del citado contrato. ${ }^{31}$ Teniendo en cuenta que su vigencia comenzó el 2 de julio de 2012 (al año de la publicación de la LCTI en el BOE), el plazo ha vencido el 2 de julio de 2014, sin que se haya cumplido con la previsión

30 Disposición adicional 18a. de la LCTI.

31 Disposición adicional 2a. de la LCTI. 
Esta revista forma parte del acervo de la Biblioteca Jurídica Virtual del Instituto de Investigaciones Jurídicas de la UNAM

normativa. Por el momento, estos nuevos contratos se rigen, en lo que no disponga la LCTI, por lo especificado en el Estatuto de los Trabajadores (ET). No obstante, es habitual que las convocatorias de ayudas para su fomento amplíen el sistema jurídico de la relación, al menos en lo que se refiere a salario o duración.

De hecho, tras la entrada en vigor de la normativa comentada, se han publicado en nuestro país, junto con otras más específicas, distintas convocatorias que sufragan los gastos salariales y de seguridad social ocasionados por la suscripción de un contrato predoctoral. Su finalidad es promover la formación en programas de doctorado de solvencia formativa e investigadora de quienes deseen orientar su actividad profesional hacia la docencia universitaria y/o a la investigación. Todas ellas son, habitualmente, muy similares, variando tan sólo en el órgano que las convoca. Algunas de sus principales características son las siguientes: ${ }^{32}$

32 Para este estudio se evalúan las siguientes convocatorias: 1) Resolución del 26 de diciembre de 2014, de la Secretaría de Estado de Educación, Formación Profesional y Universidades, por la que se convocan ayudas para la formación de profesorado universitario, de los subprogramas de Formación y de Movilidad incluidos en el Programa Estatal de Promoción del Talento y su Empleabilidad, en el marco del Plan Estatal de Investigación Científica y Técnica y de Innovación 2013-2016 (BOE, núm. 313, del 27 de diciembre de 2014); 2) Resolución del 18 noviembre de 2013, de la Secretaría de Estado de Educación, Formación Profesional y Universidades, por la que se convocan diversas ayudas para la formación de profesorado universitario de los subprogramas de Formación y de Movilidad dentro del Programa Estatal de Promoción del Talento y su Empleabilidad, en el marco del Plan Estatal de Investigación Científica y Técnica y de Innovación 2013-2016 en I+D+i (BOE, núm. 279, del 21 noviembre 2013); 3) Resolución del 5 de junio de 2015, de la Secretaría de Estado de Investigación, Desarrollo e Innovación, por la que se aprueba la convocatoria, correspondiente al 2015, de las ayudas para contratos predoctorales para la formación de doctores contemplada en el Subprograma Estatal de Formación del Programa Estatal de Promoción del Talento y su Empleabilidad, en el marco del Plan Estatal de Investigación Científica y Técnica y de Innovación 2013-2016 (BOE, núm. 140, del 12 junio 2015); 4) Resolución del 9 agosto 2013, de la Secretaría de Estado de Investigación, Desarrollo e Innovación, por la que se aprueba la convocatoria, correspondiente al 2013, de diversas actuaciones contempladas en el Subprograma Estatal de Formación y en el Subprograma Estatal de Movilidad, del Programa Estatal de Promoción del Talento y su Empleabilidad, en el marco del Plan Estatal de Investigación Científica y Técnica y de Innovación 2013-2016 (BOE, núm. 194, del 14 agosto 2013), y 5) Resolución del 4 septiembre de 2014, de la Secretaría de Estado de Investigación, Desarrollo e Innovación, por la que se aprueba la convocatoria, correspondiente al 2014, de las ayudas para contratos predoctorales para la formación de doctores contemplada en el Subprograma Estatal de Formación del Programa Estatal de Promoción del Talento y su Empleabilidad, en el marco del Plan Estatal de Investigación Científica y Técnica y de Innovación 2013-2016 (BOE, núm. 218, del 8 septiembre de 2014). 
1) El número de subvenciones en relación al de solicitantes confirma en todas ellas que la oferta es mínima, pues no permite beneficiar ni al 10\% de aquellos potenciales investigadores que la requieren. De una primera valoración cabe deducir que el interés por la investigación es muy alto, en atención a la cantidad de personas que han concurrido a la convocatoria. Si bien es cierto que, en la época de crisis que vivimos, el hecho de que más de diez mil personas se presenten a un puesto de trabajo a jornada completa, salario superior al mínimo y de una duración estimada de cuatro años, hace recapacitar acerca de si la verdadera vocación de todos los aspirantes es la investigación o, tal vez, es simplemente el hecho de obtener un trabajo. Por otra parte, en relación a la captación de los mejores científicos - en caso de suponer que el proceso de selección es el correcto- cabe aventurar que ante la gran afluencia de solicitantes se ha escogido a los mejores, pero también se puede considerar que muchos de los buenos se han quedado fuera, ya que las ayudas concedidas son, indudablemente, menos de las que se necesitan.

2) La ayuda cubre la retribución de abonar al beneficiario de la misma, el coste de las aportaciones de la cuota empresarial a la seguridad social y el de la matrícula en el programa de doctorado. Se confinan varios mecanismos de fomento de la actividad investigadora; de un lado, para el beneficiario, que además de percibir un salario se le sufragan los costes de la matrícula de la etapa doctoral, de otro lado, para la entidad en la que se adscriba el beneficiario, cuyos gastos laborales se costean completamente por la ayuda obtenida.

3) La duración máxima de este tipo de ayudas es de cuarenta y ocho meses, o setenta y dos en caso de discapacitados, descontado de ésta los periodos que el beneficiario haya disfrutado de otras becas con anterioridad o ayudas dirigidas a la formación doctoral. Por lo tanto, se sufraga el periodo máximo de un contrato predoctoral, lo que favorece la aceptación de los beneficiarios por parte de las entidades de adscripción, ya que éstos no suponen para ellas coste alguno. Asimismo, se respeta la duración del contrato establecida por la LCTI, además de que se fija una duración mínima de la subvención de veinticuatro meses, lo que en la práctica puede acarrear problemas, tanto en caso de no obtener un informe favorable en la primera de las prórrogas necesarias, con lo que el contrato habría estado vigente tan sólo doce meses; como en aquellas situaciones en las que el beneficiario, por haber disfrutado de una ayuda con la misma finalidad con anterioridad, le resten menos de esas veinticuatro 
Esta revista forma parte del acervo de la Biblioteca Jurídica Virtual del Instituto de Investigaciones Jurídicas de la UNAM

mensualidades para finalizar el periodo máximo, por lo que cabe suponer que se le denegará el beneficio.

4) Las tareas a las que el doctorando se ha de dedicar durante la vigencia del contrato son: la realización de la tesis doctoral, la participación en proyectos de investigación en los que esté incorporado y a un máximo de sesenta horas anuales de tareas docentes en caso de prestar servicios en una universidad. Cabe destacar que la LCTI ha evitado mención alguna al tiempo dedicado por estos investigadores a la docencia, cuando sí lo establece para el contrato de acceso al SECTI. La intención puede haber sido centrar este periodo en el desarrollo de la tesis, sin cargar al doctorando de tareas docentes. Sin embargo, las convocatorias surgidas con posterioridad posibilitan, como lo hacían en el régimen anterior, la asunción de carga docente por este colectivo. ${ }^{33}$

5) Actualmente, la retribución fijada para estas convocatorias es de 1.025 euros mensuales, los dos primeros años de contrato y de 1.173 el tercer y cuarto año, incrementándose en dos pagas extraordinarias anuales. La misma se ha calculado conforme los porcentajes fijados por la LCTI y con base en la categoría de ayudante, regulada en el artículo 49 de la Ley Orgánica 6/2001 de 21 de diciembre, de Universidades (LOU), ${ }^{34}$ en su redacción dada por la Ley Orgánica 4/2007 de 12 de abril. No obstante, en esta ocasión, las cuantías resultantes se han incrementado, de forma que la retribución bruta anual es equivalente a la fijada en la convocatoria 2012 de ayudas para becas y contratos de formación de profesorado universitario. Cualquier incremento o reducción en estas ayudas que se produzca con posterioridad se aplicará a todos los contratos suscritos. Asimismo, estas cantidades pueden ser incrementadas con cargo a la entidad contratante. Sin embargo, ninguna de estas cuantías hace más atractiva la carrera investigadora, como así pretende la LCTI, pues éstas se posicionan bajo la barrera del mileurismo. Incluso son cantidades inferiores a las que se obtienen en situación de beca, periodo en el cual la exención de impuestos y la cuota mínima a pagar a la seguridad social

33 Moreno Gené, J., "El contrato predoctoral...", op. cit., p. 16, considera que "a priori parece admisible que se prevea la posibilidad de que los investigadores en formación puedan colaborar en tareas docentes" ya que esta etapa le ha de servir para incorporarse a la carrera investigadora y a la carrera docente. Pese a ello, el autor opina que la LCTI ha debido establecer la finalidad formativa de esta tarea y un máximo de carga docente que le permita cumplir plenamente con el objetivo primordial de elaboración de la tesis.

34 BOE, núm. 307 del 24 diciembre de 2001. 
permiten una reducción inapreciable de la cantidad bruta asignada. Por ello, teniendo en cuenta que estamos ante trabajadores altamente cualificados, se trata de un salario irrisorio por el que cabe prever que provocará su fuga hacia otros puestos de mejores condiciones y mayores expectativas laborales.

6) La suspensión del contrato supone, para estas ayudas, una ampliación del periodo contractual, lo que no sucedía en épocas anteriores, puesto que ésta se supeditaba a la posibilidad de que existiera un excedente en los presupuestos que fijaban las ayudas. En la actualidad, las entidades de adscripción son las obligadas al pago del salario y cotización durante el tiempo en que el contrato permanezca en suspenso. Con esta medida la protección del investigador se ve claramente reforzada, pero quizás pueda ser un aspecto negativo a valorar por la entidad de destino, pues a esta le puede suponer un gasto.

7) Otro aspecto determinante para tomar la decisión de iniciarse en el campo de la investigación es la expectativa de un futuro profesional. Sobre ello no existe regulación, ni en la LCTI ni en las convocatorias de ayudas. De esta manera, una vez finalizado el contrato predoctoral, el investigador (doctor) tendrá que concurrir de nuevo a otro tipo de ayudas o concurso de méritos que le permitan suscribir un contrato de acceso al SECTI para continuar en la investigación dentro del sector público. La crisis y otros factores han provocado que en las universidades ya no sea común el paso directo del periodo de doctoramiento a un contrato con la universidad — como el de ayudante o ayudante-doctor - y, por lo tanto, la carrera investigadora en estas instituciones se vuelve cada vez más compleja, larga y exigente. Por otro lado, si lo que el investigador desea es acceder al sector privado, ha de concurrir a las pruebas selectivas que se estimen pertinentes, con la ventaja de haber obtenido el título de doctor pero con la desventaja, en muchos casos, de no haber tenido contacto con el mundo empresarial durante cuatro años. Para evitar esto, algunas ayudas apoyan la promoción en la carrera científica de aquellos beneficiarios que defiendan y aprueben su tesis en los tres primeros años de contrato. El estímulo consiste en la contratación de estos ya como doctores durante un año, percibiendo un salario mayor al que reciben durante el doctorado. En realidad, no supone un aumento de duración de la ayuda, pero sí permite obtener más méritos y, además, la retribución durante el último año sería superior. De todos modos, la medida no es suficiente a pesar de tratarse de un primer paso para robustecer la carrera investigadora, al menos, de aquellos que manifiestan una significativa dedicación a su tesis doctoral. Como 
Esta revista forma parte del acervo de la Biblioteca Jurídica Virtual del Instituto de Investigaciones Jurídicas de la UNAM

crítica, cabe pensar que esto puede discriminar a investigadores de temas que requieran un periodo amplio de tiempo para completar buenos resultados.

\section{El contrato de acceso al Sistema Español de Ciencia, Tecnología e Innovación}

Una vez finalizado el periodo formativo y obtenido el título de doctor, la siguiente etapa de la carrera investigadora ha de suponer la aplicación de lo adquirido en un puesto que conlleve una mayor autonomía. Sin embargo, evaluada la regulación del contrato de acceso al SECTI, ${ }^{35}$ se puede apreciar que este sigue siendo un contrato formativo, de similares características al contrato en prácticas.

De hecho, la LCTI establece que el trabajo que ha de desarrollar el contratado debe consistir en aquellas tareas de investigación que le proporcionen "un elevado nivel de perfeccionamiento y especialización", para así poder consolidar "su experiencia profesional". Por lo tanto, se reconoce que el doctor cuenta con cierto bagaje laboral, sin embargo, el legislador no lo considera suficiente, y razona que ha de seguir perfeccionándose. Lo dicho, lejos de ser una falsedad, puede considerarse incluso necesario, pues en toda carrera se necesita progresar y aprender continuamente. No obstante, esto no ha de suponer ninguna merma de los derechos sociales de los doctores recientes, puesto que ahora no existe justificación alguna para considerar a estos incapaces de desempeñar con total autonomía el puesto de investigador. Además, en esta etapa el contratado no pretende obtener un título, ni tampoco difiere su trabajo del que realizan los investigadores más experimentados, por lo tanto, cabría tratar a ambos de manera homogénea.

Así las cosas, la normativa establece como requisito diferenciador del contrato predoctoral, que el empleado haya obtenido el título de doctor, lo que justifica su paso a esta etapa posterior. De otra parte, la duración tampoco coincide, siendo como mínimo un año y máximo cinco, sin poder superarse en ningún caso, aún suscribiendo diferentes contratos de acceso al SECTI por distintas entidades. Las prórrogas, de haberlas, tampoco pueden concederse por periodos inferiores al año. Con ello, se insiste en el marcado carácter temporal de la carrera investigadora, pues ambos contratos estudiados suponen un total de nueve años en los que se entiende que existe una razón

35 Artículo 22 de la LCTI. 
contractual perecedera a pesar de que en la realidad las tareas no difieren con el paso de los años, salvo el título que se obtiene con la elaboración de la tesis o la temática a la que se puede dedicar el trabajador en diferentes etapas. Indudablemente, esta temporalidad no favorece la atracción y retención de talento, por lo que se debería limitar lo máximo posible o, al menos, asegurar al investigador la prolongación de su puesto en caso de que su actividad sea valorada positivamente. De hecho, a partir del segundo año el investigador (doctor) puede tener que pasar una evaluación de la que depende su continuidad en el puesto. Incluso, este sistema cabe considerarlo más duro que el de una oposición, en el que tras una sola evaluación (que puede incluir diferentes exámenes) es posible conseguir un puesto permanente, lo que no se consigue en la carrera investigadora tras una ingente cantidad de evaluaciones. Este aspecto no favorece el interés de científicos excelentes por el sector de $\mathrm{I}+\mathrm{D}+\mathrm{i}$.

Respecto a los posibles empleadores, coinciden plenamente con aquellos que se han mencionado para el contrato predoctoral, por lo tanto, en este caso también es criticable la limitación a utilizar este modelo contractual por las entidades privadas con ánimo de lucro, sobre todo cuando al doctor habría que facilitársele la incorporación al mercado de trabajo, sea en el sector público o en el privado, pues ya ha alcanzado el grado máximo en la educación reglada y, por tanto, se puede dedicar plenamente a las tareas que le encomiende la empresa.

El salario de este trabajador no se encuentra penalizado, como en la etapa anterior, pues lo contrario podría ser discriminatorio, toda vez que el trabajador ya posee la titulación y experiencia necesaria en el puesto que ha de desempeñar, lo que le permite ejercer con total capacidad. Por ello, el reducir su salario no parece que pueda tener justificación alguna.

Otro elemento que diferencia al contrato predoctoral del de acceso al SECTI es que, para este último, sí se establece la posibilidad de realizar tareas docentes, en un máximo de ochenta horas anuales. Así, si en la anterior etapa la actividad se centra en desarrollar un trabajo de investigación novedoso que conlleve a la consecución del título de doctor, en el presente periodo las labores de investigación se pueden compatibilizar con la docencia. La razón de esta distinción cabe pensar que se basa en que en la primera etapa la necesidad de formación exige que todos los esfuerzos se dirijan a investigar, pudiendo ser la docencia una distracción, pero ahora, habiendo obtenido ya una primera experiencia investigadora, se puede completar ésta con otras tareas que habi- 
Esta revista forma parte del acervo de la Biblioteca Jurídica Virtual del Instituto de Investigaciones Jurídicas de la UNAM

tualmente van ligadas, como la enseñanza. Sin embargo, a pesar de esto como ya se ha dicho, las convocatorias de ayudas a contratos predoctorales permiten igualmente la dedicación a la docencia por parte de estos trabajadores y, por lo tanto, en el fondo no existe una verdadera diferencia entre las tareas del contratado predoctoral y las del ya doctor, salvo aquellas que derivan de la temática a la que se encaminen sus estudios.

No obstante, el aspecto más discutible de la regulación de este contrato es su vinculación con el contrato en prácticas, cuyas normas son aplicables al de acceso al SECTI en todo aquello que no regule la LCTI. Con ello, se dota de un marcado carácter formativo al presente modelo contractual, similar al del contrato predoctoral, siendo este un aspecto de difícil justificación para el periodo actual. No en vano se trata de un trabajador que, como mínimo, ha estado cuatro años realizando tareas de investigación y cuyo título de doctor asegura su plena capacidad para desarrollarlas de forma autónoma. Así, este nuevo grado es el que habilita para abrir la presente etapa, siendo el mismo diferente al que ostenta el investigador para acceder al doctorado, pero ello no ha de considerarse suficiente para probar la vertiente formativa, puesto que no existe un verdadero cambio de tareas y el empleado tiene una experiencia que ha de ser valorada para considerar que lo que ahora necesita es la especialización y formación, persistentes durante toda la carrera investigadora.

Finalmente, también existen ayudas para la contratación de este tipo de personal, mas ninguna de ellas establece la obligatoriedad de utilizar el contrato de acceso al SECTI, ni siquiera lo consideran como prevalente. ${ }^{36}$ Por lo tanto, en estos casos también cabe valorar la posibilidad de suscribir un contrato por obra o servicio, un contrato en prácticas o el contrato de acceso al SECTI. Así, en caso de necesitar el puesto durante los cinco años permitidos por la norma tan sólo se puede utilizar el nuevo modelo contractual, puesto que los dos restantes no tienen una duración que permita su empleo a lo largo de toda la situación.

36 Véase resolución del 1o. de diciembre de 2014, de la Secretaría de Estado de Investigación, Desarrollo e Innovación, por la que se aprueba la convocatoria correspondiente al 2014, de diversas actuaciones contempladas en el Subprograma estatal de formación y en el Subprograma estatal de incorporación del Programa estatal de promoción del talento y su empleabilidad en el marco del Plan estatal de investigación científica y técnica de innovación 2013-2016 (BOE, núm. 294 del 5 diciembre 2014). En esta norma se regula el procedimiento de solicitud y resolución de los programas Juan de la Cierva (con una duración de dos años) y Ramón y Cajal (con una duración de cinco años). 
Esta revista forma parte del acervo de la Biblioteca Jurídica Virtual del Instituto de Investigaciones Jurídicas de la UNAM

\section{El contrato de investigador distinguido}

La última etapa de la carrera investigadora para la que la LCTI crea un nuevo modelo contractual ${ }^{37}$ es la de aquel investigador doctor que por su experiencia y méritos logrados en algún campo de estudio posee una reputación reconocida por el colectivo científico. Este personal ha de poseer el título de doctor y las tareas a las que se ha de dedicar son la realización de actividades de investigación o la dirección de equipos humanos, centros de investigación, instalaciones y programas científicos, y tecnológicos relevantes, en función de su ámbito de conocimientos.

Este contrato se caracteriza por su elevado nivel de independencia, toda vez que son las partes las que pueden fijar la mayor parte de los aspectos que regulen la relación laboral. De esta manera, tanto el salario como la duración y jornada se vinculan al acuerdo entre los interesados y al clausulado del contrato.

La única obligación del empleador para con el investigador es la necesidad de preavisarlo con tres meses de antelación en caso de querer extinguir la relación laboral, sin que esta decisión haya de justificarse por causa alguna. El incumplimiento de este periodo supone el derecho del trabajador a percibir la cuantía correspondiente al salario del periodo incumplido y la equivalente a la de una indemnización por despido improcedente fijada en el ET. ${ }^{38}$

Por lo tanto, se trata de una regulación básica del contrato, que se ha de completar mediante los acuerdos a los que lleguen el trabajador y el empresario. Esta cualidad se justifica por la posición favorable que al investigador distinguido le da su prestigio, dejándolo al nivel del empleador, lo que los faculta para poder llegar a acuerdos de manera franca, sin que ninguno de ellos ostente ventaja alguna.

\section{CONCLUSIONES}

1. Las condiciones establecidas tanto por la regulación del contrato predoctoral como por las convocatorias de ayudas que subvencionan contratos de esta modalidad hacen un poco más atractiva que en etapas anteriores la salida profesional hacia la investigación. Sin embargo, la solución no es com-

37 Artículo 23 de la LCTI.

38 Artículo 56 del ET. 
Esta revista forma parte del acervo de la Biblioteca Jurídica Virtual del Instituto de Investigaciones Jurídicas de la UNAM

pleta, puesto que existen aspectos que no mejoran, a pesar de haber dejado atrás las precarias becas formativas. Así, se sigue apostando por una contratación temporal, que si bien tiene lógica por ser una etapa formativa que no puede subsistir en el tiempo, se podría apostar por una continuación más estable en la carrera de investigación. Pero, en la realidad, una vez alcanzado el grado de doctor, los investigadores siguen luchando por contratos temporales unidos a proyectos de los que no se tiene la certeza que vayan a ser ofertados año tras año. Además, la salida profesional de un doctor se ve — en muchos casos - limitada a las universidades, puesto que, durante la etapa formativa del doctorado no se favorece la colaboración con la realidad empresarial, lo que podría darle la clave para en un futuro, acceder a un puesto en el mercado laboral privado. Para ello, sería conveniente posibilitar la utilización de este tipo contractual a los doctorados industriales que, por su naturaleza, insertan al investigador en el seno de la empresa privada.

Por otra parte, la retribución establecida tampoco puede competir con la que el potencial solicitante podría encontrar en otro puesto, incluso de menor cualificación y responsabilidad y, mucho menos, con los que se podría encontrar en el extranjero. De esta forma, la capacitación de los mejores seguirá dependiendo de una parte vocacional que haga decidir a estos talentos por lo que les gusta, renunciando a lo que les aporte una mayor retribución económica con — seguramente- mucho menos esfuerzo. Asimismo, con base en las ayudas convocadas, probablemente éstas no sean suficientes ni con una remuneración adecuada para tener un efecto notable en el fomento de la investigación. Además, a esto se le suma que en los últimos años, a causa de la crisis, aquéllas no han sido publicadas periódicamente como se venía haciendo (por ejemplo, durante 2011 y 2014 no han existido ayudas FPU) y el proceso de concesión se ha demorado hasta el punto de resolverse en el año siguiente a su aprobación, uniéndose así con la que debía ser la siguiente convocatoria.

2. Desde el momento en que el trabajador es doctor, el EPIF obligaba a su contratación laboral, pero hasta que se promulga la LCTI no se crea una modalidad contractual específica para este periodo. Con ello se pretende evitar la disparidad de las condiciones laborales que afecta a este colectivo, que en muchas ocasiones siguen siendo considerados como investigadores "de segunda", a pesar de que su titulación y experiencia aseguran su capacidad y autonomía en el puesto. Así, el contrato de acceso al SECTI tampoco es una solución completa, puesto que perpetua tres aspectos negativos que no ayudan a la 
retención del talento científico: 1) la temporalidad, posibilitando que durante cinco años puedan estar contratados de manera temporal, sin que tampoco al finalizar este plazo se les asegure la continuidad en el puesto; 2) la sensación de seguir siendo un colectivo en formación, sobre todo, por su vinculación al contrato en prácticas y la impresión de que esa necesidad de perfeccionamiento y especialización es única para este periodo, cuando en realidad toda la carrera investigadora, por la innovación que posee implícita, está dotada de esta característica. Por lo tanto, se trata de una distinción en cuanto a la posterior carrera investigadora, un tanto artificiosa, pues el trabajador realiza las mismas funciones que los científicos en etapas siguientes, y 3) el distanciamiento con las entidades privadas con ánimo de lucro, apartando al investigador del mercado laboral privado, lo que puede suponer una oportunidad de futuro para este colectivo.

No obstante, como aspecto positivo cabe destacar la retribución que no se penaliza en comparación al personal investigador que realice actividades análogas, y de lo que se puede deducir que los investigadores doctores así contratados poseen la misma capacidad que otros cuyas funciones sean similares.

3. Para una etapa avanzada de la carrera investigadora, la LCTI crea el contrato de investigador distinguido, cuya característica principal es la flexibilidad de los actores para regular sus condiciones laborales. La única obligación que asume el empleador es la necesidad de preavisar su deseo de extinguir la relación laboral, lo cual conlleva una indemnización a favor del trabajador equivalente a la de un despido improcedente.

4. La Ley contiene, además, un avance muy favorable, pues obliga la contratación laboral de todos aquellos beneficiarios de ayudas a la investigación que impliquen la realización de tareas de $\mathrm{I}+\mathrm{D}+\mathrm{i}$, tanto por las empresas públicas como las privadas. Con ello se reduce al mínimo la posibilidad de seguir apoyándose en becas con una mínima cobertura social para vincular a estos trabajadores con las diferentes entidades.

5. En el ámbito universitario, la LOU establece modalidades contractuales específicas que se adhieren con las fijadas por la LCTI. Así, los contratos de ayudante, profesor-ayudante, doctor y profesor visitante son equivalentes al contrato predoctoral, el de acceso al SECTI y el de investigador distinguido, respectivamente. Por ello, y con el fin de simplificar al máximo el mapa contractual de nuestro país, cabe valorar la eliminación de duplicidades y, por tanto, prescindir de las categorías citadas que se regulan en la LOU, puesto 
que las reguladas por la LCTI también se dirigen, principalmente, al ámbito de las universidades.

6. La regulación que contiene la LCTI es muy básica, puesto que la norma obliga al gobierno a elaborar un Estatuto que desarrolle estas modalidades contractuales. Cabe destacar que esta premisa está siendo incumplida desde junio de 2012, por lo que es necesario primar esta regulación que posiblemente solvente muchos de los problemas que el vacío legal o la supletoriedad de las normas puede estar provocando.

7. Además de las condiciones laborales que atraen y retienen al personal investigador, todas estas medidas han de ir acompañadas de un esfuerzo económico por parte de la administración que fomente y promueva la inversión en $\mathrm{I}+\mathrm{D}+\mathrm{i}$, necesario para que éste se convierta en un sector principal de la economía del país. Ty 